\title{
Comparison of the success rate of intraosseous infusion performed by Japanese paramedics
}

Kenichi KIUCHI 1,3), Kyutaro KAWAGISHI 2), Ryo SAGISAKA ${ }^{3)}$, Hideharu TANAKA ${ }^{3)}$

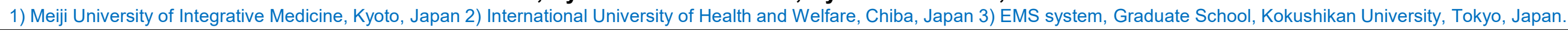

\section{BACKGROUND}

Quick access and large bore fluid replacement to the shock trauma victim is a critical component of resuscitation.

However, Japanese paramedics are not yet available to perform intraosseous infusion (IOI) right now and only performing periphera intravenous access is allowed. Previous survey showed more than $2 / 3$ of the out of hospital cardiac arrest (OHCA) patients could not receive peripheral intra-venous access by paramedics in Japan.

\section{PURPOSE}

We investigated the knowledge and the technical problems of Japanese paramedics performing IOI.

\section{METHODS}

Under the approval of the Ethics Committee this study, Ten experienced Japanese paramedics with more than 5 years of were recruited, attempted $\mathrm{IOI}$ insertion to the both side of the proximal tibia and the humeral head of cadavers. Cadavers were donated for the medical education and researches at the Shinshu university school of medicine.

For the IOI needle insertion, EZ-IO (Wayne, PA, USA) was used in this study. Before inserting needle to the cadaver, 30 minutes of lecture was given to the paramedics.

The insertion site for the proximal tibia, the tibial tuberosity was set as a landmark, and two finger width inferior and one finger width medial to the landmark was set as an insertion site.

The insertion site for the humeral head, the coracoid process was set as a landmark, and two finger width lateral to the landmark was set as an insertion site.

The times to detect landmarks and total times of $\mathrm{IOI}$ (from landmark detection to start infusion) were measured and compared. In addition, success rate of the IOI were also compared.
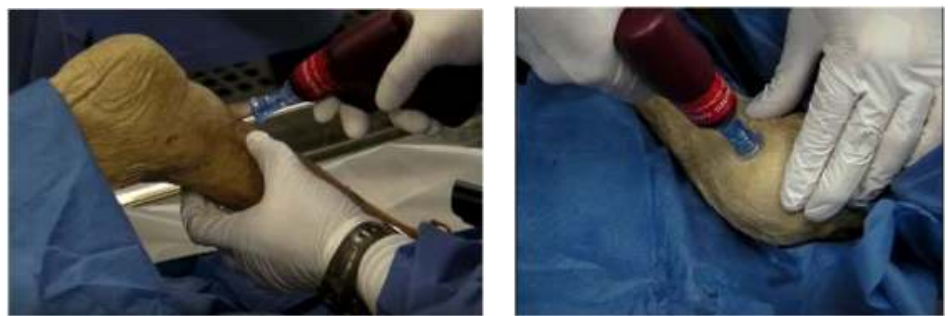

Figure shows insertion of IOI needle to the proximal tibia (Left) and to the humeral head (Right) of the cadaver, by EZ-IO.

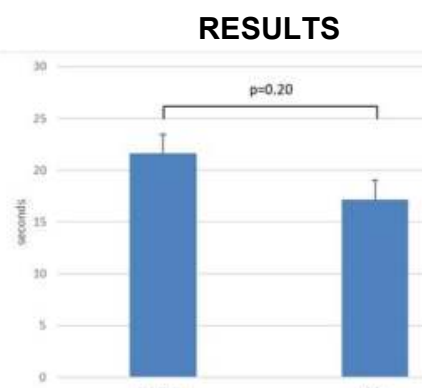

The times to detect the landmark of the insertion site were $17.2 \pm 1.8$ seconds for the proximal tibia and $21.6 \pm 1.8$ seconds for the humeral head.

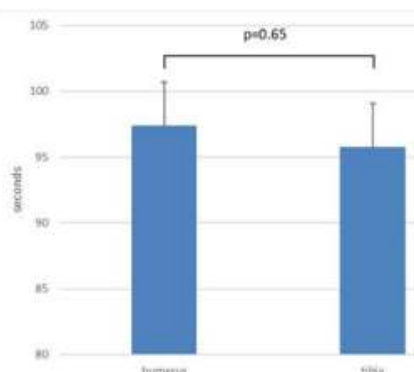

The total times for IOI needle insertion was $95.7 \pm 3.3$ seconds to the proximal tibia and $97.4 \pm 3.3$ seconds to the humeral head.

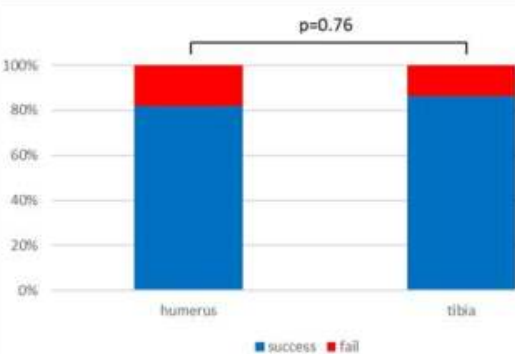

The success rates of $\mathrm{IOI}$ were $85 \%$ at the proximal tibia and $80 \%$ at the humeral head.

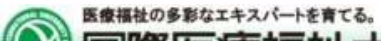

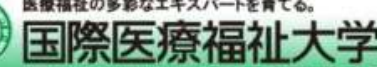

\section{DISCUSSION}

In our study, there were no significant differences in the time of detecting landmarks and the total time of $\mathrm{IOI}$ between the proximal tibia and the humeral head. Also, there was no significant differences in success rate of IOI between the proximal tibia and the humeral head.

However, success rate of $\mathrm{IOI}$ was slightly higher at the proximal tibia in our study. Furthermore, time to detect the landmark and total time of $\mathrm{IOI}$ were faster at the proximal tibia

In previous study of comparing $\mathrm{IOI}$ insertion devices (Andrzej Kurowski et al. Am J of Emerg Med. 32 (2014) 1490-1493), success rate of EZ-IO was reported as $82.66 \%$ by polish paramedics. Other report of EZ-IO (Brett Gendron et al. Mil Med. 183(5-6), (2018) e216-e222) concluded that the success rate of $\mathrm{IOI}$ by U.S. army medics was $88 \%$ at the tibia and $86 \%$ at the humerus.

If we compare our results with those studies, Japanese paramedics showed similar success rate and proved that they have enough ability to perform IOI.

\section{CONCLUSION}

We considered that $\mathrm{IOI}$ is reliable method to establish infusion route in case of emergency, even for Japanese paramedics.

We assumed that the proximal tibia is relatively safer and higher success rate to establish $\mathrm{IOI}$ route because it is easy to detect the anatomical landmark, if there are no contradictions.

We hope Japanese government will open scope of practice to Japanese paramedics to perform IOI in the nearest future.

will also visit the same time next year.

\section{Acknowledge}

We would like to thank for all donors of the cadavers and all paramedics who have participated in this study. 